\title{
Impact of an Information Leaflet on Knowledge of Hepatocellular Carcinoma and Hepatitis B among Chinese Youth
}

\author{
Jun-Jie Ouyang ${ }^{1}$, Wen-Jing He ${ }^{2}$, Kai-Xin Zheng ${ }^{1}$, Geng-Zhen Chen ${ }^{3 *}$
}

\begin{abstract}
Background: To assess the effect of an information leaflet on the level of Chinese youth's knowledge about hepatitis B and hepatocellular carcinoma (HCC), the most common type of primary liver cancer (PLC). Materials and Methods: A total of 500 students, from two universities in the Chaoshan area of China, were randomly divided into an intervention group of 280 participants and a control group of 220 . Baseline knowledge of HCC and hepatitis B was evaluated by questionnaire interview. Subsequently, only the intervention group was given an information leaflet of $\mathrm{HCC}$ and hepatitis $\mathrm{B}$. Three months later, the two groups were contacted for a second interview. Changes in knowledge from baseline of $\mathrm{HCC}$ and hepatitis B were compared between the two groups. Results: There was no statistically significant difference in mean PRE-questionnaire scores between the intervention and control groups. However, the mean POST-questionnaire score was significantly higher in the intervention group after the intervention. The leaflet had the greatest effect on the participants' questionnaire score, and raised their level of knowledge about HCC and hepatitis B. Conclusions: The information leaflet intervention is significantly effective in improving the knowledge of HCC and hepatitis B among the youth.
\end{abstract}

Keywords: Primary liver cancer - hepatocellular carcinoma - hepatitis B - information leaflet - knowledge

Asian Pac J Cancer Prev, 17 (1), 439-443

\section{Introduction}

Primary liver cancer is the sixth most common cancer (748,000 cases in 2008) and the third most frequent cause of death from cancer $(696,000$ deaths in 2008) in the world (Ferlay et al., 2010; Jemal et al., 2011; Sheng-Long, 2012). China accounts for about $50 \%$ of these cases and deaths worldwide (Jemal et al., 2011). According to estimates, liver cancer is the fourth most common cancer, accounting for $11.6 \%$ of all new cancer cases in China, and is the second most fatal cancer with $15.97 \%$ of total cancer deaths (Wei et al., 2014). HCC is not the most widespread cancer, but is the second most deadly malignant tumor in the Chaoshan area of China (Qin et al., 2010). Hence, $\mathrm{HCC}$ has been a serious public health problem in the area where the study was performed.

Representative risk factors for development of HCC include cirrhosis, hepatitis $\mathrm{C}$ virus (HCV), aflatoxin, alcohol consumption, tobacco smoking, dietary factors, obesity and diabetes (Chuang et al., 2009), with epidemiological research showing the most significant risk factor being chronic infection of hepatitis B virus (HBV), which causes more than half of the HCC cases worldwide (Parkin, 2006). According to statistics, one third of chronic
HBV infection occurs in China (Xu and Wang, 2009). A health survey launched in Shantou city in 2009 indicates that the positive incidence of HBsAg accounts for $8.1 \%$ of primary and secondary school students (Zhang et al., 2011). Therefore, the younger generation is a high risk population for HBV infections.

As the age of patients with malignancy gradually gets younger (Huang et al., 2006), youth represents a key population of becoming potential HCC sufferers (Kao and Chen, 2005). This younger generation may be more vulnerable to risk factors and hostile environments. Consequently, the prevention of $\mathrm{HCC}$ and hepatitis $\mathrm{B}$ among the youth is extremely important. Due to the environmental cause of HCC infection, effective prevention can be achieved by a high level of knowledge and awareness, leading to avoidance of risk factors (Fatohy et al., 1998; Mahdy et al., 1998). A pilot study on knowledge about HCC and hepatitis B among inpatients of the Chaoshan area has reveals that the general population lacks adequate knowledge of these diseases (He et al., 2013). This result suggests a strong need for carrying out an HCC prevention campaign to improve knowledge and awareness. In view of this situation, an educational intervention of information leaflet may provide effective 
improvement of knowledge and awareness, as the leaflet is designed to be easily understood and kept by the target population (Humphris et al., 1999).

The aim of our present study is to evaluate the effect of an HCC and hepatitis B information leaflet on knowledge about these diseases among the youth in universities where no HCC prevention campaign had been implemented previously. The result of this study can become a reference resource for schools or health authorities to direct comprehensive cancer prevention campaign among the youth, thereby reducing the incidence and mortality of HCC and hepatitis B.

\section{Materials and Methods}

\section{Participants and methods}

This study was performed between June and September of 2014 in two universities in the Chaoshan area. An intervention group of 280 participants and a control group of 220 participants were randomly selected from different majors in these universities.

The study consisted of two interviews separated by a time interval of three months. Every participant was first interviewed by a medical practitioner to record students' answers on the questionnaire (PRE) regarding HCC and hepatitis B. At the end of the interview, only the participants of intervention group were given the information leaflet. Three months later, each one of the participants was interviewed by a medical practitioner to complete the questionnaire (POST) a second time. Finally, only participants who pointed out the same demographic data in the PRE and the POST questionnaires were eligible for the study.

\section{Informed consent}

Before the interview, each participant was informed about the objectives of the study and the confidentiality of participant's information. This study was approved by the ethics committee of Shantou University Medical College.

\section{Questionnaire}

The questionnaire was modified from the original version used in the previous study by He et al. (2013), to include demographic data and the participant's knowledge regarding $\mathrm{HCC}$ and hepatitis B. Demographic data encompassed gender, age, place of residence, university grade, and major. The participant's knowledge regarding HCC and hepatitis B was divided into six series: risk factors of HCC (16 items), symptoms and signs of HCC (12 items), prevention methods for HCC (10 items), treatment methods for HCC (6 items), transmission routes of hepatitis B (6 items) and prevention methods for hepatitis B (5 items).

\section{Leaflet}

The leaflet was designed by the researchers after consulting professional texts and materials of related diseases and was validated by professors of internal medicine and epidemiology (Figure 1). The leaflet contained three parts: (a) title: information on prevention of liver cancer and hepatitis B, (b) description and epidemiology of HCC, (c) description of hepatitis B. There were about 400 words in the leaflet along with pictures about the risk factors for HCC and the transmission routes of hepatitis B. It was printed on an A4 size paper.

Before the study, 10 university students were asked to read the leaflet in order to ensure the leaflet was comprehensible. Each student read the leaflet for ten minutes and then was asked to describe the content of the leaflet. The misunderstandings were corrected according to the students' suggestions.

\section{Statistical analysis}

Participants of both groups were asked to answer 55 questions from the same questionnaire in the two interviews and their final scores were recorded. A correct response was given a score of 1 while an incorrect response was given a score of 0 , so that the total score ranged from 0 to 55. Data were analyzed with SPSS software (version 19.0). A chi-square test was performed on the demographic data. A T-test was performed on the differences between the knowledge of the intervention group and the control group. Multiple regression analysis was performed to analyze the effect of the intervention on knowledge of HCC and hepatitis B, and the relationship between knowledge and variables such as information leaflet and demographic data.

\section{Results}

There were 280 participants in the intervention group and 220 participants in the control group for the first interview. Three months later, 278 participants from the intervention group and 214 participants from the control group were re-contacted. A total of 263 intervention group participants admitted reading the information leaflet and were considered reliable enough for the second interview. After excluding the unqualified questionnaires, 259 and 212 questionnaires were included in the final analysis. The response rate was 92.5 (259/280) and $96.36(212 / 220)$, respectively.

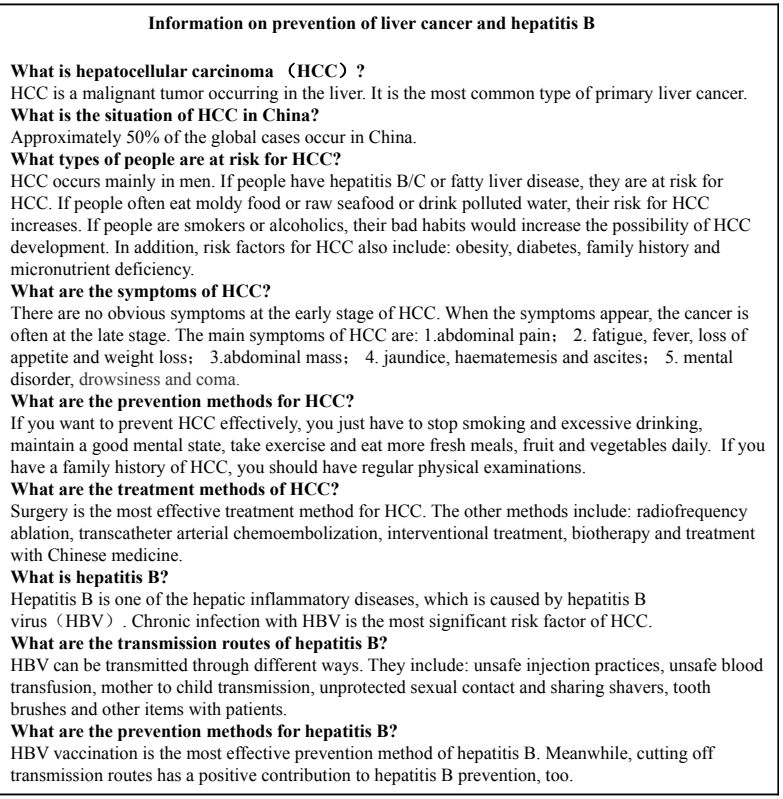

Figure 1. Information Leaflet (Original Version is in Chinese and Pictures are not Displayed) 
Table 1. Demographic Characteristics of Participants

\begin{tabular}{|c|c|c|c|c|}
\hline Characteristics & $\begin{array}{l}\text { Intervention group } \\
\mathrm{N}=259(\%)\end{array}$ & $\begin{array}{c}\text { Control group } \\
\mathrm{N}=212(\%)\end{array}$ & Test statistic value $\left(\chi^{2}\right)$ & $P$-value \\
\hline \multicolumn{5}{|l|}{ Gender } \\
\hline Male & $139(53.7)$ & $108(50.9)$ & 0.347 & 0.556 \\
\hline Female & $120(46.3)$ & $104(49.1)$ & & \\
\hline \multicolumn{5}{|l|}{ Age } \\
\hline $17 \sim 19$ & $64(24.7)$ & $46(21.7)$ & 4.93 & 0.085 \\
\hline $20 \sim 22$ & $166(64.1)$ & $153(72.2)$ & & \\
\hline $23 \sim 25$ & $29(11.2)$ & $13(6.1)$ & & \\
\hline \multicolumn{5}{|l|}{ Resident place } \\
\hline Urban & $142(54.8)$ & $115(54.2)$ & 0.016 & 0.9 \\
\hline Suburban & $117(45.2)$ & $97(45.8)$ & & \\
\hline \multicolumn{5}{|l|}{ Major } \\
\hline Medical & $44(17.0)$ & $27(12.7)$ & 1.647 & 0.199 \\
\hline Non-medical & $215(83.0)$ & $185(87.3)$ & & \\
\hline
\end{tabular}

Table 2. Mean Values (Standard Deviations) of PRE-Questionnaire Score, POST-Questionnaire Score and Score Improvement

\begin{tabular}{|c|c|c|c|c|}
\hline Score & Intervention group & Control group & Test statistic value $(\mathrm{T})$ & $P$-value \\
\hline \multicolumn{5}{|l|}{ Total } \\
\hline PRE score & $29.74(9.07)$ & $31.26(9.61)$ & -1.769 & 0.078 \\
\hline POST score & $49.83(6.71)$ & $34.74(9.13)$ & 20.043 & $<0.0001$ \\
\hline POST-PRE & $20.10(10.18)$ & $3.47(8.76)$ & 19.044 & $<0.0001$ \\
\hline \multicolumn{5}{|c|}{ Risk factors of HCC } \\
\hline PRE score & $7.51(3.18)$ & $7.93(3.52)$ & -1.357 & 0.175 \\
\hline POST score & $14.35(2.66)$ & $9.34(3.31)$ & 17.81 & $<0.0001$ \\
\hline POST-PRE & $6.84(3.62)$ & $1.42(3.79)$ & 15.844 & $<0.0001$ \\
\hline \multicolumn{5}{|c|}{ Symptoms and signs of HCC } \\
\hline PRE score & $5.52(3.50)$ & $5.80(3.73)$ & -0.853 & 0.394 \\
\hline POST score & $11.11(2.06)$ & $6.92(3.51)$ & 15.368 & $<0.0001$ \\
\hline POST-PRE & $5.59(3.66)$ & $1.12(3.88)$ & 12.845 & $<0.0001$ \\
\hline \multicolumn{5}{|c|}{ Prevention of $\mathrm{HCC}$} \\
\hline PRE score & $8.47(2.38)$ & $8.80(2.19)$ & -1.536 & 0.125 \\
\hline POST score & $9.81(0.89)$ & $9.00(1.92)$ & 5.619 & $<0.0001$ \\
\hline POST-PRE & $1.33(2.46)$ & $0.20(2.57)$ & 4.857 & $<0.0001$ \\
\hline \multicolumn{5}{|c|}{ Treatment of HCC } \\
\hline PRE score & $2.58(1.33)$ & $2.80(1.39)$ & -1.733 & 0.084 \\
\hline POST score & $5.49(1.13)$ & $3.30(1.48)$ & 17.701 & $<0.0001$ \\
\hline POST-PRE & $2.91(1.63)$ & $0.50(1.43)$ & 16.826 & $<0.0001$ \\
\hline \multicolumn{5}{|c|}{ Transmission of hepatitis B } \\
\hline PRE score & $2.61(1.78)$ & $2.75(1.78)$ & -0.871 & 0.384 \\
\hline POST score & $5.05(1.34)$ & $2.89(1.78)$ & 14.613 & $<0.0001$ \\
\hline POST-PRE & $2.44(2.17)$ & $0.14(1.96)$ & 12.115 & $<0.0001$ \\
\hline \multicolumn{5}{|c|}{ Prevention of hepatitis B } \\
\hline PRE score & $3.05(1.12)$ & $3.18(1.09)$ & -1.305 & 0.192 \\
\hline POST score & $4.03(0.62)$ & $3.28(1.02)$ & 9.423 & $<0.0001$ \\
\hline POST-PRE & $0.98(1.20)$ & $0.09(1.20)$ & 7.954 & $<0.0001$ \\
\hline
\end{tabular}

Table 3. Stepwise Multiple Regression Analysis

\begin{tabular}{clccccc}
\hline Model & Standard regression equation & $\mathrm{R}$ & $\mathrm{R} 2$ & Adjusted R2 & $\mathrm{F}$ & $P$ \\
\hline 1 & $\mathrm{Y}=15.1 \mathrm{X}_{1}+19.6$ & $0.690^{\mathrm{a}}$ & 0.476 & 0.475 & 426.5 & $<0.0001$ \\
2 & $\mathrm{Y}=15.1 \mathrm{X}_{1}+1.8 \mathrm{X}_{2}+16.9$ & $0.695^{\mathrm{b}}$ & 0.483 & 0.481 & 218.7 & $<0.0001$ \\
3 & $\mathrm{Y}=15.1 \mathrm{X}_{1}+1.7 \mathrm{X}_{2}+2.2 \mathrm{X}_{3}+14.6$ & $0.699^{\mathrm{c}}$ & 0.489 & 0.485 & 148.7 & $<0.0001$ \\
\hline
\end{tabular}

aPredictors: $\mathrm{X}_{1}=$ leaflet; ${ }^{\text {PPredictors: }} \mathrm{X}_{1}=$ leaflet, $\mathrm{X}_{2}=$ gender; ${ }^{\text {PPredictors: }} \mathrm{X}_{1}=$ leaflet, $\mathrm{X}_{2}=$ gender, $\mathrm{X}_{3}=$ major; ${ }^{\mathrm{d}}$ Dependent Variable: $\mathrm{Y}=$ total score

\section{Demographics}

Demographic data of the participants is shown in Table 1. The intervention and control groups were comparable in terms of gender, age, place of residence, and major. The differences of all these demographic data were not statistically significant between the two groups $(P>0.05)$. More than $80 \%$ of participants in both groups were non- medical students.

PRE-interventional knowledge and POST-interventional knowledge

The differences between the two groups' knowledge are summarized in Table 2. At baseline, the level of knowledge for HCC and hepatitis B were similar 
between intervention group and control group with the mean PRE-questionnaire total scores of the two groups being 29.74 and 31.26 points $(P>0.05)$, respectively. At follow-up, the mean POST-questionnaire total score of intervention group rose by 20 points to 49.83 (out a total of 55) points after the intervention, while a change of only 3.47 points was found in the control group's total score. There was a statistically significant difference between the mean POST-questionnaire total score of the two groups $(P<0.0001)$. This showed that the knowledge of the intervention group improved after the leaflet intervention.

The mean PRE-questionnaire scores between the two groups were similar in every part of the questionnaire $(P>0.05)$. However, the POST-questionnaire score of the intervention group was significantly higher than the control group's score in each part of the questionnaire $(P<0.0001)$. These results further support the above conclusion that knowledge of HCC was improved by the intervention..

\section{The relationship between knowledge and variables}

The relationship between knowledge and variables was investigated by performing step-wise multiple regression analysis. In order to analyze the effect of the intervention on HCC and hepatitis B knowledge, the POST-questionnaire score was used as the dependent variable. On the other hand, predictors as independent variable were dichotomized or trisected so that a low score was given to the potential situation of risk. The predictors contained: leaflet (control group, score 1; intervention group, score 2), gender (male, score 1; female, score 2), age (17 19 years, score 1; 20 22 years, score 2; 23 25 years, score 3 ), place of residence (suburban, score 1; urban, score 2), major (non-medical, score 1; medical, score 2). Through the analysis, the statistically significant predictors were included in the final model.

As shown in Table 3, the information leaflet was the most significant predictor to affect the POST-questionnaire score (Model 1), accounting for $47.6 \%$ of the variability in the total score $(\mathrm{R} 2=0.476)$. This suggested that the leaflet was the most effective driving force in improving HCC and hepatitis B knowledge. The two other significant predictors were gender (Model 2) and major (Model 3), revealing that females or medical students have a higher level of HCC and hepatitis B knowledge than their counterparts. However, from Model 1 to Model 2, adding gender only increased the variability by $0.7 \%$; from Model 2 to Model 3, adding the major only raised the variation of the total score by $0.6 \%$. Therefore, gender and major ultimately had only a minimal effect on the total score compared with the impact of the leaflet.

\section{Discussion}

Studies on the prevention of HCC are rare and this study is the first evaluation of HCC and hepatitis B educational intervention in the Chaoshan area, where HCC mortality is high (Qin et al., 2010) and the general knowledge of HCC and hepatitis B is low (He et al., 2013). In our present study, poor knowledge of HCC and hepatitis B among the youth was confirmed by the
PRE-questionnaire. Upon follow-up, intervention by information leaflet distribution effectively raised the knowledge of HCC and hepatitis B. In addition, our study revealed the present situation of $\mathrm{HCC}$ and hepatitis $\mathrm{B}$ knowledge among university students.

The younger generation has a growing risk of HCC and it is a challenge for them to make the right decisions to prevent this disease. The incidence of HCC can be dramatically reduced if the people are made aware of its risk factors, such as hepatitis B (Robotin et al., 2014). Performing educational intervention of a cancers, associated with known risk factors, among people should raise prevention awareness by elevating knowledge, leading to avoidance behavior (Moralez et al., 2012; Quadri et al., 2014; Abiodun et al., 2014). Therefore, the dissemination of knowledge, beneficial to the public's health, should be at the forefront of intervention. In our study, the leaflet intervention elevated knowledge of HCC and hepatitis B. This result represents an effort to contribute to existing and future research on health issues of HCC and hepatitis B. On the basis of our study, the government can utilize information leaflets as a form of health educational intervention to promote prevention, thereby achieving the goal of reducing the incidence and mortality of high prevalent diseases.

Our study has demonstrated a positive effect of information leaflet distribution. Interestingly, the control group's mean POST-questionnaire score still rose by 3.47 points above the mean PRE-questionnaire score without the leaflet, though the change was small. This phenomenon might be explained by that the result of the second interview could be affected by the first interview. After the first interview, the control group's participants might proactively search for answers to some of the questionnaire questions that were of interest. However, compared to the improvement following intervention, the improvement of the control group is scarcely noticeable $(P<0.0001)$.

The strength of our study is the high response rate of using the information leaflet. The intervention group was studied to deal with two questions: (1) would the leaflet be used by the participants, and (2) what was the effect of the leaflet among the participants who read it? Hence, it was unnecessary to give a second interview to participants who did not read the leaflet. In this study, about $94 \%$ of intervention group participants $(263 / 280)$ claimed that they read the leaflet during the three-month period. About $98 \%$ of these participants $(259 / 263)$ were available for the final analysis. The data indicates that the participants are willing to accept the educational intervention of the information leaflet. This high response rate could ensure the reliability of this study.

One limitation of our study is the limited target population. The study was just carried out in two universities among the students who were 17- to 25-years old. Thus, due to potential sampling bias, the results of the study may not be representative of the entire population. Furthermore, university students represent the highly educated population who should have a higher capacity to accept and understand the information leaflet. Consequently, our findings may not comprehensively 
Impact of an Information Leaflet on Knowledge of Hepatocellular Carcinoma and Hepatitis B among Chinese Youth

represent the effectiveness of the leaflet among people of different classes, while the younger people and those who are more educated may benefit more from the intervention (Petti and Scully, 2007). In view of this, future studies should select a more comprehensive sample to evaluate the effect of the information leaflet.

The second limitation of the study is the absence of the change on participants' attitude and behavior. A positive health-related attitude and behavior may impel people to stay away from risk factors and seek a health examination regularly (Torabi and Seffrin, 1989; Fatohy et al., 1998). This would help in preventing the diseases or treating the disease at an early stage when there is a hope for better prognosis. Our study reveals that the information leaflet intervention can be useful in improving the participants' knowledge of HCC and hepatitis B. However, whether such intervention will change their health-related attitude and behavior is unknown. Future studies should focus on evaluating the aforementioned issues.

Cancer is a global public health concern, but many countries have not given adequate attention to its prevention. China, which accounts for the most cases of HCC and hepatitis B worldwide, has not had a corresponding prevention project. Hence, the public health organization has a responsibility to work out an effective and comprehensive prevention program for HCC. Health authorities can utilize the information leaflet as a form of health educational intervention to impart knowledge of prevention to the youth or other people. Meanwhile, other forms of health educational intervention should also be developed. The interaction of these interventions may provide more contribution to the prevention effort.

In summary, our study shows that the intervention by an information leaflet can improve the knowledge of HCC and hepatitis B among the youth. The results demonstrated the improvement of knowledge of: risk factors, symptoms and signs, prevention methods and treatment methods of $\mathrm{HCC}$, and transmission routes and prevention methods of hepatitis B. As a result, this study provids direction for further public health initiatives in this high prevalent area. Some effective HCC and hepatitis B educational programs can be formulated and carried out in the future. On the other hand, due to the limitations of our study, future large-scale studies should design a comprehensive scale to evaluate the information leaflet and confirm that its efficiency can benefit more people.

\section{Acknowledgements}

The authors want to thank all the participants who took part in this study. The study was not subsidized by any grants and its cost was borne by the authors. The work should belong to the Department of Minimally Invasive Surgery, Second Affiliated Hospital of Shantou University of Medical College.

\section{References}

Abiodun OA, Olu-Abiodun OO, Sotunsa JO, Oluwole FA(2014). Impact of health education intervention on knowledge and perception of cervical cancer and cervical screening uptake among adult women in rural communities in Nigeria. $B M C$ Public Health, 14, 814.

Chuang SC, La Vecchia C, Boffetta $P$ (2009). Liver cancer: Descriptive epidemiology and risk factors other than HBV and HCV infection. Cancer Letters, 286, 9-14.

Ferlay J, Shin HR, Bray F, et al (2010). Estimates of worldwide burden of cancer in 2008: GLOBOCAN 2008. Int J Cancer, 127, 2893-917.

Fatohy IM, Mounir GM, Mahdy NH, El-Deghedi BM (1998). Improving students' knowledge, attitude and practice towards cancer prevention through a health education program. Part II. J Eqypt Public Health Assoc, 73, 755-85.

Huang MX, Zhong FX, Guo SH, Xu HY (2006). Statistical analysis of the low age tendency of malignant tumor. Chin $J$ Postgrad Med, 29, 53-4.

He WJ, Xu MY, Xu RR, et al (2013). Inpatients' Knowledge about Primary Liver Cancer and Hepatitis. Asian Pac J Cancer Prev, 14, 4913-8.

Humphris GM, Duncalf M, Holt D, Field EA (1999). The experimental evaluation of an oral cancer information leaflet. Oral Oncol, 35, 575-82.

Jemal A, Bray F, Center MM, et al (2011). Global Cancer Statistics. CA Cancer J Clin, 61, 69-90.

Kao JH, Chen DS (2005). Changing disease burden of hepatocellular carcinoma in the Far East and Southeast Asia. Liver Int, 25, 696-703.

Mahdy NH, Fatohy IM, Mounir GM, El-Deghedi BM (1998). Assessment of students' knowledge, attitude and practice concerning cancer and its prevention. Part I. J Eqypt Public Health Assoc, 73, 399-431.

Moralez EA, Rao SP, Livaudais JC, Thompson B (2012). Improving knowledge and screening for colorectal cancer among Hispanics: overcoming barriers through a PROMOTORA-led home-based educational intervention. J Cancer Educ, 27, 533-9.

Parkin DM (2006). The global health burden of infectionassociated cancers in the year 2002. Int J Cancer, 118, 3030-44.

Petti S, Scully C (2007). Oral cancer knowledge and awareness: Primary and secondary effects of an information leaflet. Oral Oncol, 43, 408-15.

Qin SL, Lin YC, Li CT, Xie XL (2010). Statistical analysis of inpatients with malignancies and death rate in shantou cancer hospital from 2004 to 2008. IMHGN, 16, 889-92.

Quadri MF, Saleh SM, Alsanosy R, et al (2014). Effectiveness of an intervention program on knowledge of oral cancer among the youth of Jazan, Saudi Arabia. Asian Pac J Cancer Prev, 15, 1913-8.

Robotin MC, Kansil MQ, Porwal M, et al (2014). Communitybased prevention of hepatitis-B-related liver cancer: Australian insights. Bull WHO, 92, 374-9.

Sheng-Long Y (2012). Asia-Pacific Primary Liver Cancer Expert Meetings. Liver Cancer, 1, 61.

Torabi MR, Seffrin JR (1989). Evaluation of the effects of cancer education on knowledge, attitude, and behavior of university undergraduate students. J Cancer Educ, 4, 39-47.

Wei KR, Yu X, Zheng RS, et al (2014). Incidence and mortality of liver cancer in China, 2010. Chin J Cancer, 33, 388-94.

Xu YY, Wang JY (2009). Hepatitis B, Hepatocirrhosis and Liver Cancer. J Clin Intern Med, 26, 728-30.

Zhang LL, Lin JX, Zhang JH (2011). The health survey of primary and secondary school students in Shantou. Chin J School Doctor, 25, 557-8. 